\title{
SEMES: Um Sistema Educacional Móvel para o Ensino de Engenharia de Software
}

\author{
Nemésio Freitas Duarte Filho ${ }^{1}$, Gabriel Antônio Tavares Conrado ${ }^{1}$, Henrique \\ Forioni de Lima ${ }^{1}$, Ellen Francine Barbosa ${ }^{1}$ \\ ${ }^{1}$ Instituto de Ciências Matemáticas e de Computação (ICMC/USP) - Universidade de \\ São Paulo. Caixa Postal 13.566-590 - São Carlos - SP - Brasil \\ \{nemesio,francine\} @icmc.usp.br, \{gabaconrado, henriquelhfl\}@gmail.com
}

\begin{abstract}
Mobile learning has been used in various areas of knowledge, flexibilizing the support and dissemination of knowledge. The learning in the field of computing, along with their sub-themes may be supported by mobile learning. In this context, this work presents the development of a mobile learning system with a focus in the discipline of Software Engineering. The educational content of the system was modeled following the AIM-CID approach, and evaluated by specialists and users. The experimental results demonstrate that the system has a structured layout, ensuring greater usability, cognition and facility of learning by the apprentices.

Resumo. A aprendizagem móvel vem sendo utilizada em diversas áreas do conhecimento, flexibilizando o apoio e a disseminação de conhecimento. $O$ ensino na área da Computação, juntamente com os seus sub-temas, podem ser apoiados pela aprendizagem móvel. Neste contexto, este trabalho discute o desenvolvimento de um sistema educacional móvel com foco na disciplina de Engenharia de Software, denominado SEMES. O conteúdo educacional do sistema foi modelado seguindo a abordagem AIM-CID, sendo avaliado por especialistas e usuários. Os resultados experimentais demostram que o sistema apresenta um layout bem estruturado, garantindo maior usabilidade, cognição e facilidade de aprendizagem por parte dos aprendizes.
\end{abstract}

\section{Introdução}

Sistemas e ambientes de aprendizagem têm apresentado uma crescente importância nos últimos anos, tendo um papel fundamental no ensino e treinamento, sendo relevantes não apenas no ambiente acadêmico, mas também no meio industrial (Keegan, 2005; Crompton, 2013).

Esses sistemas, juntamente com o advento e desenvolvimento da computação ubíqua, vêm proporcionando um novo modelo de aprendizagem, a aprendizagem móvel (m-learning). Este novo modelo proporciona uma maior motivação e interação entre os aprendizes e instrutores, podendo eles contribuir, participar e acessar o sistema de ensino por meio de dispositivos móveis, tais como celulares, i-pods, laptops, rádio, telefone, fax, entre outros, a qualquer momento e em qualquer lugar (Kukulska-Hulme and Traxler, 2005).

Muitos sistemas e aplicativos educacionais móveis estão sendo desenvolvidos para apoiar o aluno no aprendizado de temas nas mais variadas áreas de conhecimento, 
entre elas, a Computação. Este apoio educacional tem proporcionado maior motivação e interatividade para os aprendizes, além de possibilitar maior flexibilidade no ensino (Kinshuk et al., 2005; Kukulska-Hulme and Traxler, 2005).

Dentro da perspectiva apresentada, este artigo discute a proposta de desenvolvimento do SEMES - um sistema de aprendizagem móvel para o ensino de Engenharia de Software. O conteúdo didático associado ao sistema foi modelado com a ajuda de especialistas da área, a partir da utilização da abordagem AIM-CID (Abordagem Integrada de Modelagem - Conceitual, Instrucional e Didática) (Barbosa and Maldonado, 2011).

Pretende-se, com isso, contribuir para a melhoria e qualificação acadêmica dos aprendizes na área de Computação e, mais especificamente, em Engenharia de Software. De fato, a Engenharia de Software a cada ano vem ganhando mais importância. No entanto, ainda há resistência de pessoas e organizações em sua aplicação, sobretudo em virtude da falta de informações sobre o tema (Ghosh, 2006). Assim, conhecer e entender os fundamentos que norteiam a área é um aspecto importante para a efetiva adoção das práticas de Engenharia de Software no cenário de produção de software. O desenvolvimento de sistemas e aplicações educacionais móveis sobre Engenharia de Software insere-se nesse contexto, visando contribuir para o domínio e a disseminação do conhecimento na área.

$\mathrm{O}$ artigo encontra-se organizado como se segue. A Seção 2 apresenta a base teórica sobre aprendizagem móvel e modelagem de conteúdos educacionais. A Seção 3 traz uma visão geral do sistema SEMES, descrevendo aspectos de seu desenvolvimento e principais funcionalidades. A Seção 4 apresenta a avaliação do sistema proposto. Por fim, a Seção 5 apresenta as conclusões e perspectivas para trabalhos futuros.

\section{Referencial Teórico}

\subsection{Aprendizagem Móvel (m-learning)}

Conceitualmente o m-learning pode ser definido como qualquer tipo de ensino ou treinamento que ocorre quando um aprendiz não está em um lugar predeterminado ou fixo, ou quando o indivíduo aproveita-se das oportunidades de aprendizagem proporcionadas pelos dispositivos móveis (Moura, 2009; Crompton, 2013).

Por se tratar de um paradigma novo e emergente, há diversas tentativas para definir m-learning. Rachid and Ishitani (2012) definem m-learning como uma atividade que permite aos indivíduos serem mais produtivos quando consomem, criam ou interagem com as informações, mediados por dispositivos digitais móveis e portáteis, que acompanham o indivíduo de forma regular, desde o início ao fim das tarefas. Ozdamli and Cavus (2011) abordam m-learning como uma atividade que permite aos indivíduos serem mais produtivos quando consomem, criam ou interagem com as informações, mediados por dispositivos digitais móveis e portáteis.

Este novo paradigma de ensino, além de facilitar a aprendizagem de seus usuários, pode também ser utilizado no treinamento corporativo. Assim, os usuários podem passar mais tempo onde necessitam estar, dedicando seu tempo a atividades de atualização de seu conhecimento e a uma possível formação continuada (Koschembahr, 2005). Por outro lado, os sistemas de m-learning também podem apresentar limitações de uso como, por exemplo: poder de processamento reduzido, tela de tamanho variável, energia limitada (dependente de baterias), comunicação com taxas de transmissão, 
geralmente, menores do que as das redes fixas, aspectos de usabilidade, entre outras (Pachler, 2007).

Por meio dos benefícios proporcionados pela aprendizagem móvel, já é possível observar e identificar iniciativas na literatura abordando sua utilização na área da Computação, flexibilizando o ensino e a capacitação de aprendizes. A aprendizagem móvel aplicada diretamente em Computação deve proporcionar a melhora da disseminação do conhecimento, principalmente em relação a temas mais abstratos e teóricos.

\subsection{Abordagem Integrada de Modelagem - Conceitual, Instrucional e Didática (AIM-CID)}

A abordagem AIM-CID (Abordagem Integrada de Modelagem - Conceitual, Instrucional e Didática) caracteriza-se por um conjunto de modelos genéricos (Modelo Conceitual, Modelo Instrucional e Modelo Didático) para a representação de conteúdos educacionais (Barbosa and Maldonado, 2011). Cada modelo aborda aspectos distintos da atividade de modelagem. A Figura 1 sintetiza os principais aspectos da abordagem AIM-CID.

- O modelo conceitual consiste em uma descrição de alto-nível do domínio de conhecimento que se deseja ensinar.

- O modelo instrucional é responsável pela definição de informações adicionais relativas aos conceitos previamente identificados. Estas informações caracterizam-se por exemplos, dicas, sugestões de estudo, exercícios, simulações, entre outras.

- Por fim, o modelo didático é responsável por associar os objetos anteriormente modelados, estabelecendo uma sequência de apresentação entre eles.



Figura 1. Abordagem AIM-CID (Barbosa and Maldonado, 2011).

Os conteúdos educacionais associados ao sistema de aprendizagem móvel deste trabalho foram modelados em conformidade com a abordagem AIM-CID, sendo desenvolvidos de forma integrada e interativa. Todo o desenvolvimento foi realizado com o apoio de especialistas da área. Os detalhes e exemplos da construção e elaboração destes módulos educacionais serão apresentados nas próximas seções. 


\section{SEMES: Visão Geral}

O sistema desenvolvido neste trabalho é um sistema de aprendizagem móvel, cujo objetivo é promover formação continuada aos aprendizes que buscam adquirir conhecimentos sobre o tema de Engenharia de Software, contribuindo assim para a melhoria e qualificação acadêmica dos cursos de Computação bem como para a disseminação do conhecimento em geral. Os usuários em sua maioria são graduandos (jovens entre 18 e 30 anos), sendo também possível a utilização do SEMES por profissionais da área.

\subsection{Processo de Desenvolvimento}

O modelo do processo de desenvolvimento foi definido nas seguintes etapas:

1. Concepção e Definição do Escopo: a definição da problemática surgiu a partir das necessidades e carências encontradas na comunidade e no meio científico. A área de Computação apresenta muitos temas que tendem a ser abstratos e teóricos para os aprendizes. Especificamente com o tema Engenharia de Software, verificou-se uma carência em relação a um sistema de apoio ao ensino, capaz de melhorar a formação dos profissionais da área, facilitando o entendimento e disseminação de conhecimento.

2. Estudo da abordagem AIM-CID: com ênfase no entendimento de seus modelos associados (Conceitual, Instrucional e Didático), inicialmente foi realizada a modelagem dos conteúdos educacionais em conformidade com os modelos da abordagem AIM-CID. A título de ilustração, a Figura 2 apresenta o modelo didático elaborado sobre o tema "Modelos de Processos de Software - Prototipação". Ressaltase que antes de implementar os módulos educacionais os modelos educacionais foram revisados com a ajuda de especialistas da área.

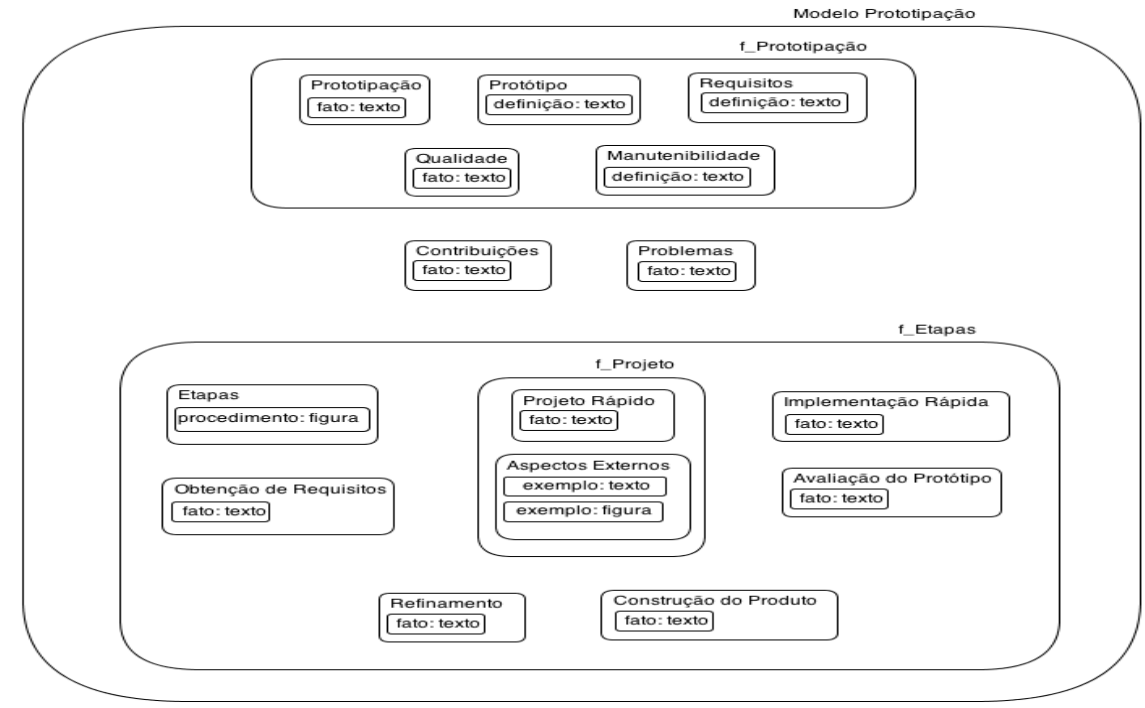

Figura 2. Modelo Didático referente ao tema "Modelos de Processo de Software (Prototipação)".

3. Identificação e estudo de tecnologias para o desenvolvimento de aplicações móveis: verificando as necessidades de construção de um aplicativo educacional móvel, algumas tecnologias foram estudadas para a identificação das mais relevantes e eficiente ao problema em questão. Para este fim, a construção do protótipo foi realizada com base no sistema operacional Android para dispositivos móveis, tendo em vista o conhecimento e flexibilidade dos pesquisadores no presente trabalho. 
4. Identificação e seleção dos tópicos de ensino a serem explorados: o sistema foi elaborado incorporando temas específicos da disciplina de Engenharia de Software, levando em consideração os seguintes módulos de ensino: (1) Introdução à Engenharia de Software; (2) Modelo Cascata; (3) Modelo Prototipação; (4) Modelo Incremental; (5) Modelo Espiral; e (6) Padrões de Projeto de Software. Ressalta-se que módulos adicionais estão sendo desenvolvidos e modelados garantindo a continuidade do sistema, sendo incorporados e testados futuramente.

5. Elaboração dos materiais didáticos digitais e sua aplicação no contexto de disciplinas de graduação: antes da elaboração do sistema, requisitos foram levantados e identificados, proporcionando um sistema direcionado para as práticas educacionais. Através de consultas em trabalhos relacionados à aprendizagem móvel e demonstrações de sistemas prontos, foram levantados os requisitos funcionais necessários para um aplicativo de aprendizagem móvel.

6. Avaliação: para evidenciar os resultados finais, foram realizados testes com especialistas da área e usuários comuns (alunos e aprendizes), possibilitando a identificação de limitações e possíveis melhorias no protótipo.

\subsection{Principais Características e Funcionalidades}

O SEMES foi implementado procurando incorporar interações de usabilidade, visando maior flexibilidade frente aos usuários do sistema, sendo elas:

- Conteúdo: apenas o conteúdo necessário é apresentado para o usuário, evitando que informações desnecessárias sejam disponibilizadas;

- Número de Clicks: uma das premissas para aplicações de m-learning é reduzir o número de clicks por partes dos usuários. Assim, optou-se por atribuir no máximo dois clicks para os usuários mudarem de páginas ou de layout;

- Plano de Fundo: evitou-se utilizar figuras no plano de fundo, sendo utilizadas apenas cores simples, procurando com isso garantir a atratividade para os usuários do sistema;

- Contraste e Disposição dos elementos: com base nos critérios do CRAP (Contraste, Repetição, Alinhamento e Proximidade) (Nielsen, 1993), foi possível definir um layout mais flexível aos usuários do sistema;

- Botões grandes: a utilização de botões maiores em dispositivos móveis é uma premissa importante, já que o usuário utilizará mecanismos táteis;

- Botões de Atalho (Help/Close/Back): foram inseridos botões de atalho, com o objetivo de facilitar a interação com os usuários. Mesmo sendo redundantes, usuários já familiarizados com aplicações desktop e web sentem a necessidade de botões básicos visíveis, sendo eles: help, back e close.

Com estes critérios de usabilidade, o sistema proporciona aos aprendizes e tutores maior facilidade e flexibilidade em relação à utilização dos conteúdos educacionais. As principais funcionalidades do sistema (Figura 3) foram estruturadas da seguinte maneira:

- Menu principal (Figura 3.a): menu em forma de lista, com todas as funcionalidades do sistema. 
- Menu de curso (Figura 3.b): informações do curso, com título e um resumo com os principais assuntos abordados; Menu em forma de lista, com os tópicos e subtópicos que o curso aborda.

- Tela com o conteúdo do curso (Figura 3.c): no centro da tela encontra-se o conteúdo. Na extremidade inferior há o controle de fluxo, com o indicador de páginas e controles básicos para mudá-las.

- Menu lateral de navegação (Figura 3.d): contém os tópicos da aula.

- Botões "Saiba Mais" (Figura 3.e): com informações adicionais do slide; e Botão "Dicionário" com a definição das principais palavras e termos utilizados.

- Funcionalidades Auxiliares (Figura 3.f): zoom via multitoque nas imagens dos slides; Palavras-chave linkadas ao texto, que indicam ao usuário a existência de conteúdo adicional (exemplos, comentários, definições de terminologias, entre outros).

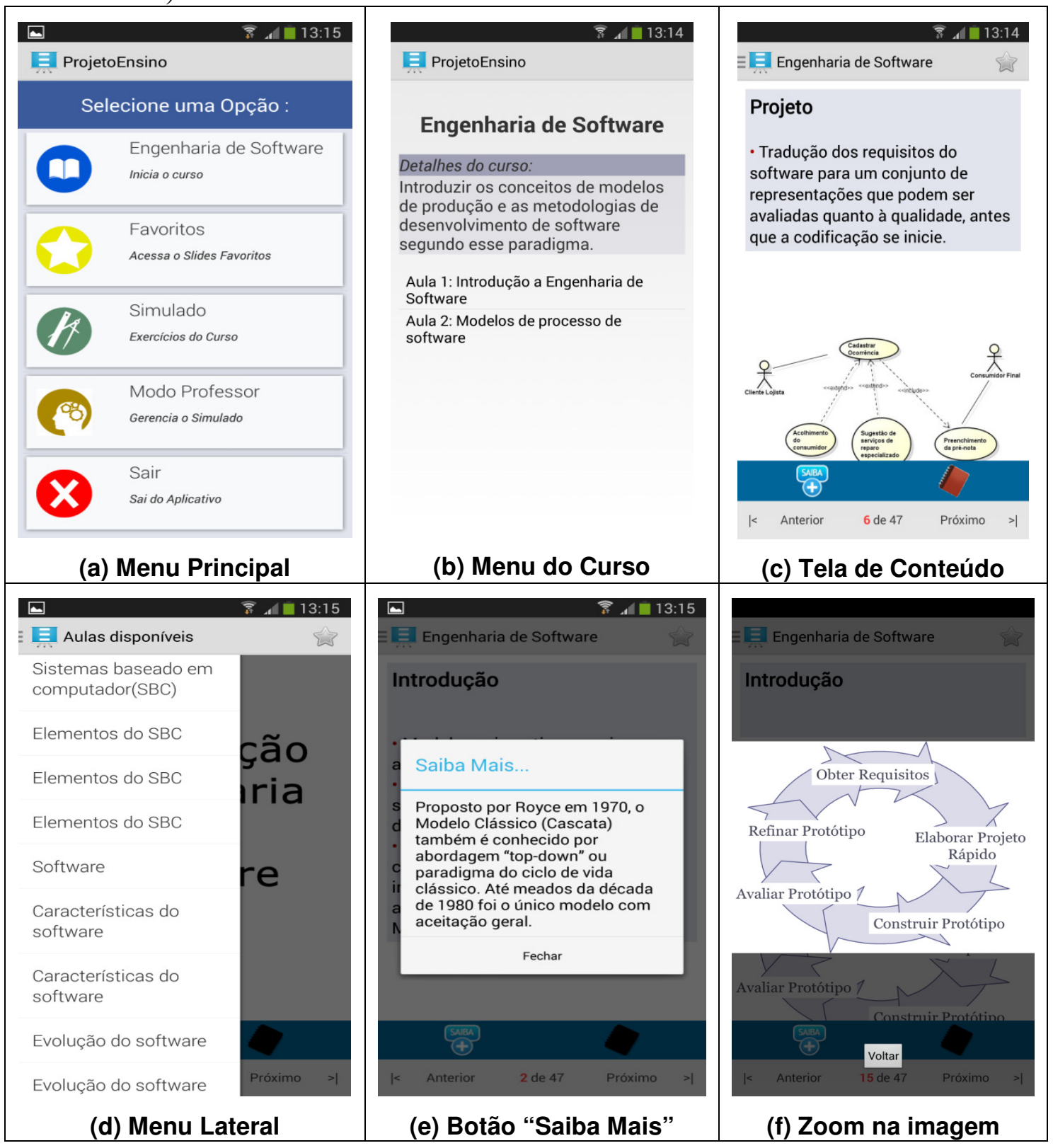


Ainda como parte do sistema SEMES, também foi desenvolvido um módulo de Simulado, com questões e testes associados aos temas de aula. O objetivo é avaliar e fornecer feedback aos alunos, além de facilitar o estudo e a fixação dos conceitos. Basicamente, a ideia é que após o término de cada aula seja aplicado o simulado, com perguntas do conteúdo recém-visto. A Figura 4 ilustra a tela de um simulado.


Figura 4. Exemplo de uma questão do simulado.

\section{Avaliação}

Esta seção apresenta uma avaliação preliminar do sistema SEMES. Para a avaliação, foram utilizados alunos do curso tecnológico de Sistemas para Internet e professores especialistas na área de Engenharia de Software. O feedback dos participantes é relevante para fornecer e proporcionar melhorias que podem ser incorporadas em versões subsequentes do sistema educacional, verificando pontos positivos e limitações educacionais.

Em um primeiro momento, um grupo de 20 alunos receberam atividades a serem realizadas no sistema SEMES. Todas as atividades realizadas com os alunos foram instruídas e supervisionadas por um tutor, que tinha na ocasião o objetivo de dar continuidade ao processo de avaliação e observar o comportamento dos alunos ao longo da realização das atividades. Os alunos realizaram as seguintes tarefas:

1. Acessar o sistema SMES: nesta atividade foi avaliado a facilidade de acesso do sistema em diferentes dispositivos móveis (com sistema operacional Android). Os alunos tiveram à disposição diferentes dispositivos, podendo também acessar as informações com o seu próprio aparelho móvel. Nenhum treinamento prévio foi realizado aos alunos, sendo definido somente o local de acesso do repositório do sistema (link). Questões de instalação e utilização também fizeram parte da avaliação e foram de responsabilidade dos alunos participantes.

2. Iniciar e finalizar um módulo do curso: ao longo do sistema, existem diferentes módulos educacionais relacionados a área de Engenharia de Software. Os alunos foram instruídos para escolherem um dos módulos e realizar a sua execução do início ao fim, passando por todos os slides e funcionalidades. 
3. Realizar um simulado relacionado ao módulo estudado: uma parte importante da avaliação de um sistema educacional é a fixação do conhecimento por parte dos aprendizes. Nesta etapa, os alunos realizaram uma bateria de questões referentes ao módulo que foi executado/estudado na etapa anterior, além do critério de fixação do conhecimento. Outros critérios também foram avaliados, sendo eles: feedback das questões, organização, estrutura, pontuações, entre outros.

4. Responder um questionário final: ao final da execução das tarefas, os alunos responderam a um questionário online sobre diversos critérios do sistema SEMES, tendo como opções de respostas as afirmativas: Muito satisfatório, Satisfatório, Regular, e Insatisfatório.

O questionário online foi aplicado ao término da realização das atividades, sendo composto por critérios (Acesso, Módulo Educacional, Simulado e Informações Adicionais). Para cada critério foram incorporadas questões de múltipla escolha para facilitar o seu preenchimento. A Tabela 1 apresenta os resultados obtidos juntamente com o número de itens e critérios de avaliação. Além disso, ressalta-se que as informações adicionais referem-se a questões complementares e campos editáveis (perguntas abertas), que permitem aos participantes colocaram pontos positivos, negativos e o que acharem necessários para contribuírem com a avaliação. Nas tabelas 1 e 2 a sua pontuação é apresentada como NA (Não se aplica).

Tabela 1. Resultado questionário com alunos.

\begin{tabular}{|l|c|c|c|c|}
\hline \multicolumn{1}{|c|}{ Critério } & $\begin{array}{c}\text { Muito } \\
\text { satisfatório }\end{array}$ & Satisfatório & Regular & Insatisfatório \\
\hline Acesso (7 itens) & $78 \%$ & $20 \%$ & $0 \%$ & $2 \%$ \\
\hline Módulo Educacional (6 itens) & $86,2 \%$ & $10 \%$ & $2,3 \%$ & $1,5 \%$ \\
\hline Simulado (6 itens) & $90 \%$ & $9 \%$ & $1 \%$ & $0 \%$ \\
\hline Informações Adicionais (3 itens) & NA & NA & NA & NA \\
\hline
\end{tabular}

Além da participação dos alunos, em um processo de avaliação de um sistema educacional é importante ter a visão crítica do professor (educador da área), no caso, da área de Engenharia de Software. O procedimento aplicado aos professores foi semelhante ao aplicado aos alunos, porém, com atividades e questões diferenciadas. Ao final, foi realizado o preenchimento de um formulário online. Este procedimento foi realizado com a participação de 5 professores. Destaca-se que os professores participantes nesta avaliação são especificamente da área de Engenharia de Software, não tendo participado da elaboração do conteúdo didático do sistema, sendo professores de instituições distintas. A Tabela 2 sintetiza os resultados obtidos pela avaliação dos professores.

Tabela 2. Resultado questionário com professores da área.

\begin{tabular}{|l|c|c|c|c|}
\hline \multicolumn{1}{|c|}{ Critério } & $\begin{array}{c}\text { Muito } \\
\text { satisfatório }\end{array}$ & Satisfatório & Regular & Insatisfatório \\
\hline Estrutura e Organização (7 itens) & $74,4 \%$ & $20 \%$ & $5,6 \%$ & $0 \%$ \\
\hline Conteúdo Educacional (8 itens) & $76 \%$ & $22 \%$ & $2 \%$ & $0 \%$ \\
\hline Inserção de Conteúdo (5 itens) & $68 \%$ & $30 \%$ & $0 \%$ & $2 \%$ \\
\hline Informações Adicionais (3 itens) & NA & NA & NA & NA \\
\hline
\end{tabular}


De modo geral, é possível concluir que o sistema SEMES apresenta uma boa aceitação tanto para alunos quanto professores, possuindo níveis considerados aceitáveis frente a critérios de acesso, utilização, conteúdo, feedback de informações, gerenciamento, entre outros aspectos. $\mathrm{O}$ critério de informações adicionais possibilitou uma visão crítica e complementar dos participantes; tanto alunos quanto professores tiveram pontos semelhantes em relação a aspectos de: (1) ausência de módulos educacionais adicionais sobre a área de Engenharia de Software, tais como gestão de projetos, requisitos, teste de software, entre outros; (2) ausência de um tutorial auto explicativo (animação), que pudesse ajudar leigos na utilização e acesso de sistemas móveis; (3) ausência de versões do sistema para outros sistemas operacionais móveis, tais como iOS e Windows Phone. Ressalta-se que neste primeiro momento o sistema SEMES foi desenvolvido com alguns módulos educacionais; outros módulos estão sendo modelados e implementados por estudantes e pesquisadores. A ausência de um tutorial auto explicativo torna-se relevante e fundamental, juntamente com a portabilidade frente a outros sistemas operacionais móveis. Tais itens serão analisados e aplicados nas próximas versões do sistema.

\section{Conclusão e Trabalhos Futuros}

No contexto da aprendizagem móvel verifica-se uma carência e precariedade de ferramentas simples e flexíveis, que proporcionem uma aprendizagem adequada por meio de dispositivos móveis e que, ao mesmo tempo, não sejam impactadas pelas limitações desses dispositivos. Assim, este trabalho teve como objetivo o desenvolvimento de um sistema educacional móvel para o ensino de Engenharia de Software, denominado SEMES.

Os conteúdos educacionais associados ao SEMES foram modelados e organizados por meio da abordagem AIM-CID, com o auxílio de especialistas da área, havendo uma preocupação na parte de conteúdo e estrutura. Esta modelagem, integrada a um sistema de aprendizagem, facilita o entendimento por parte dos aprendizes, principalmente em relação a temas abstratos e teóricos, como é o caso da Engenharia de Software.

Em relação aos pontos positivos, o sistema apresenta um layout bem estruturado, garantindo maior usabilidade, cognição e facilidade de uso por parte dos usuários. Os pontos limitantes identificados e analisados ao longo das avaliações de modo geral foram: (1) ausência módulos educacionais adicionais sobre a área de Engenharia de Software; (2) ausência de um tutorial auto explicativo; e (3) ausência de versões do sistema para outros sistemas operacionais móveis.

Como trabalhos futuros, pretende-se modificar e evoluir o protótipo com a adição de novos módulos educacionais e adição de aspectos multimídia como animações, vídeos e som. Além disso, pretende-se realizar, em curto prazo, um experimento do sistema juntamente com graduandos do curso de Computação e outros cursos correlatos, possibilitando coletar informações sobre a efetividade de aprendizagem proporcionada pelo sistema em ambientes reais de ensino e treinamento. Assim, a ideia é, permitir a formação continuada aos aprendizes, contribuindo para a melhoria e qualificação acadêmica dos cursos de Computação. 


\section{Agradecimentos}

Agradecemos à FAPESP, CAPES e CNPq pelo auxílio financeiro concedido para a realização deste trabalho.

\section{Referências}

Barbosa, E. F. and Maldonado, J. C. Collaborative development of educationalmodules: a need for lifelong learning. In Magoulas, G. D., editor,E-Infrastructures and Technologies for Lifelong Learning: Next Generation Environments, pages 175-211. IGI Global, Birkbeck College, University of London, UK. 2011.

Ghosh, R. A. Economic impact of open source software on innovation and the competitiveness of the information and communication technologies (ICT) sector in the EU. Maastricht: UNU-MERIT, p. 287. 2006.

Keegan, D. The Incorporation of Mobile Learning into Mainstream Education and Training. In: Proccedings of mLearning2005-4th World Conference on m-learning, Cape Town, 2005.

Kinshuk, T., Suhonen, J., Sutinen, E., Goh, T. Mobile technologies in support of distance learning. In:Asian Journal of Distance Education, v. 1, n. 1, p. 60 -68, 2003.

Crompton, H. Mobile learning: New approach, new theory. In Z. L. Berge \& L. Y. Muilenburg (Eds.), Handbook of mobile learning (pp. 47-57). Florence, KY: Routledge. (Awarded the 2014 Association for Educational Communications and Technology (AECT). 2013.

Koschembahr, C. Mobile Learning: the next evolution. Chief Learning Officer, February. 2005.

Kukulska-Hulme, A., Traxler, J. Mobile teaching and learning. In: Js Mobile teaching and learning. p. 25-44, 2005.

Moura, A. Geração Móvel: um ambiente de aprendizagem suportado por tecnologias móveis para a Geração Polegar. In P. Dias, A. J. Osório (Org.) Actas da VI Conferência Internacional de TIC na Educação Challenges 2009 / Desafios 2009. Braga: Universidade do Minho, p. 50-78. 2009.

Nielsen, J. Usability Engineering. 1ed. Boston: Academic Press, 1 vol. 1993.

Ozdamli, F., Cavus, N. Basic elements and characteristics of mobile learning. World Conference on Educational Technology Researches, Volume 28, p 937-94. 2011.

Pachler, N. Mobile learning: towards a research agenda. Londres. Institute of Education, University of London. 2007.

Rachid, L. C., Ishitani, L. M-tutorial: ferramenta de autoria para desenvolvimento de tutoriais voltados para o m-learning. In: Revista Brasileira de Informática na Educação (RBIE), v.20, n.1. 2012. 